\title{
A CHARACTERISATION OF LIPSCHITZ CLASSES ON 0-DIMENSIONAL GROUPS
}

\author{
WALTER R. BLOOM
}

ABSTRACT. This paper is concerned with characterising, in terms of certain properties of their Fourier transforms, the Lipschitz functions of order $a(0<a<1)$ defined on a locally compact metric 0 dimensional Abelian group.

1. Introduction. Let $G$ be a locally compact metric 0 -dimensional Abelian group with translation-invariant metric $d$. Its character group will be denoted by $\Gamma$.

We shall characterise the Lipschitz functions of order $\alpha(0<a<1)$ in terms of certain properties of their Fourier transforms. The results obtained are analogues of classical results due to Jackson and Bernstein (see [5, Chapter 3, Theorems (13.6), (13.20), respectively]).

2. Notation and preliminary results. Choose any strictly-decreasing sequence $\left(\beta_{n}\right)$ of positive numbers smaller than 1 for which there exists $\mu \in(0,1)$ such that $\beta_{n+1} \leq \mu \beta_{n}$ for all $n \in\{1,2, \ldots\}$. Consider the open sets

$$
V_{n}=\left\{x \in G: d(x, 0)<\beta_{n}\right\}
$$

Since $\left\{V_{n}\right\}_{n=1}^{\infty}$ is an open basis at 0 , it follows from $[3,(7.7)]$ that all but at most a finite number of the $V_{n}$ are contained in a compact subgroup of $G$. By deleting some of the $\beta_{n}$ if necessary, we can assume that all the $V_{n}$ enjoy this property.

Now put $\boldsymbol{\Upsilon}_{n}=A\left(\Gamma, V_{n}\right)$ (the annihilator of $V_{n}$ in $\Gamma$ ). Then each $\boldsymbol{\Upsilon}_{n}$ is a compact open subgroup of $\Gamma$, and $\boldsymbol{\Upsilon}_{1} \subset \boldsymbol{\Upsilon}_{2} \subset \ldots$. Furthermore, since (by $[3,(24.18)]$ ) every element of $\Gamma$ lies in a compact subgroup of $\Gamma$, it follows that $\Gamma=\bigcup_{n=1}^{\infty} \mathbf{\Upsilon}_{n}$.

Let $\lambda$ denote a chosen Haar measure on $G$. The spectrum (written $\Sigma(f))$ of $f \in L^{\infty}(G)$ will be defined as in [3, (40.21)]. For $f \in L^{p}(G)(p \in$ $[1, \infty))$, we define its spectrum by

$$
\Sigma(f)=\bigcup\left\{\Sigma(\phi * f): \phi \in C_{00}(G)\right\}
$$

(where $C_{00}(G)$ denotes the space of continuous functions on $G$ with compact support). We shall write

Received by the editors September 3, 1974.

AMS (MOS) subject classifications (1970). Pri mary 43A15, 43 A70. 


$$
L_{\Upsilon}^{p}(G)=\left\{f \in L^{p}(G): \Sigma(f) \subset \Upsilon\right\}
$$

where $\boldsymbol{Y}$ is any subset of $\Gamma$.

Given $f \in L^{p}(G)$, its best $p$ th power approximation by members of $L_{\boldsymbol{\Upsilon}_{n}}^{p}(G)$ is written as

$$
E_{n}(p ; f)=\inf \left\{\|f-t\|_{p}: t \in L_{\Upsilon_{n}}^{p}(G)\right\}
$$

and its mean modulus of continuity with exponent $p$ is given by

$$
\omega(p ; f ; \delta)=\sup \left\{\left\|\tau_{a} f-f\right\|_{p}: d(a, 0) \leq \delta\right\}
$$

(where $\tau_{a} f: x \rightarrow f(x-a)$ ). We shall define

$$
\operatorname{Lip}_{p} \alpha=\left\{f \in L^{p}(G): \omega(p ; f ; \delta)=O\left(\delta^{\alpha}\right)\right\},
$$

where $\alpha>0$. In the case $p=\infty$ it will be further assumed that such $f$ are continuous (and hence uniformly continuous).

Lemma. For every $f \in L^{p}(G)$ there exists $t^{*} \in L_{\mathbf{r}_{n}}^{p}(G)$ such that the infimum in (i) is attained.

Proof We can choose a sequence $\left(t_{m}\right) \subset L_{\Upsilon_{n}}^{p}(G)$ which, for each $m \epsilon$ $\{1,2, \ldots\}$, satisfies $\left\|j-t_{m}\right\|_{p} \leq E_{n}(p ; f)+m^{-1}$. Then

$$
\left\|t_{m}\right\|_{p} \leq E_{n}(p ; f)+m^{-1}+\|f\|_{p} \leq M
$$

for some $M$ independent of $m$. We now consider two cases:

(a) $1<p \leq \infty$. The ball $\left\{g \in L^{p}(G):\|g\|_{p} \leq M\right\}$ is weak ${ }^{*}$-compact in $L^{p}(G)$, and hence there exists $t^{*} \in L^{p}(G)$ and a subsequence $\left(t_{m(k)}\right)$ of $\left(t_{m}\right)$ for which $\int_{G} t_{m(k)} h d \lambda \rightarrow \int_{G} t^{*} h d \lambda$ for all $h \in L^{p^{\prime}}(G)$ (where $p^{\prime}$ is the exponent conjugate to $p$, that is, $p^{-1}+p^{\prime-1}=1$ for $p \neq \infty$, and $\infty^{\prime}=1$ ).

Choose any $h \in L_{\boldsymbol{r}_{n}^{c}}^{p^{\prime}}(G)$. Then, given $\epsilon>0$, there exists $k_{0}$ such that for all $k \geq k_{0},\left|\int_{G} t_{m(k)} h^{n} d \lambda-\int_{G} t^{*} h d \lambda\right|<\epsilon$. Now $\Sigma\left(t_{m(k)}\right) \subset \boldsymbol{\Upsilon}_{n}$ and $\Sigma(h \vee)$ $=-\Sigma(h) \subset \boldsymbol{\Upsilon}_{n}^{c}$ (where $\left.h_{\vee}: x \rightarrow h(-x)\right)$; consequently $\Sigma\left(t_{m(k)} * h \vee \subset \Sigma\left(t_{m(k)}\right)\right.$ $\cap \Sigma\left(b_{V}\right)$ is void (this inclusion follows from [3, (40.21)] and the (easily proved) property of the spectrum that $\Sigma(f)=\bigcup\left\{\Sigma(\phi * f): \phi \in C_{00}(G)\right\}$ is valid for all $f \in L^{\infty}(G)$ ). Hence

$$
\int_{G}{ }^{t}{ }_{m(k)} h d \lambda=t_{m(k)} * h \bigvee(0)=0 .
$$

Thus $\left|\int_{G} t^{*} h d \lambda\right|<\epsilon$ and, since $\epsilon>0$ was chosen arbitrarily,

$$
t^{*} * h_{\vee}(0)=\left|\int_{G} t^{*} h d \lambda\right|=0
$$

Now this holds for all $h \in L_{\mathbf{\Upsilon}_{n}^{c}}^{p^{\prime}}(G)$, whence it follows that $\Sigma\left(t^{*}\right) \subset \boldsymbol{\Upsilon}_{n}$. 
We now show that $t^{*}$ fulfills the requirement of the Lemma. For $h \epsilon$ $L^{p^{\prime}}(G),\|h\|_{p^{\prime}} \leq 1$, and $\epsilon>0$ given we can find $k_{0}$ such that

$$
\max \left\{m\left(k_{0}\right)^{-1},\left|\int_{G} t_{m\left(k_{0}\right)} h d \lambda-\int_{G} t^{*} b d \lambda\right|\right\} \leq \epsilon \text {. }
$$

Then

$$
\begin{aligned}
\left|\int_{G} f h d \lambda-\int_{G} t^{*} h d \lambda\right| \leq & \left|\int_{G} f h d \lambda-\int_{G}{ }^{t}{ }_{m\left(k_{0}\right)} h d \lambda\right| \\
& +\left|\int_{G} t_{m\left(k_{0}\right)} h d \lambda-\int_{G} t^{*} h d \lambda\right| \\
& \leq\left\|f-t_{m\left(k_{0}\right)}\right\|_{p}+\epsilon \leq E_{n}(p ; f)+2 \epsilon .
\end{aligned}
$$

As this holds for all $\epsilon>0$, we must have

$$
\left|\int_{G} f h d \lambda-\int_{G} t^{*} h d \lambda\right| \leq E_{n}(p ; f)
$$

and

$$
\left\|f-t^{*}\right\|_{p}=\sup \left\{\left|\int_{G} f h d \lambda-\int_{G} t^{*} h d \lambda\right|:\|h\|_{p^{\prime}} \leq 1\right\} \leq E_{n}(p ; f) .
$$

The reverse inequality is trivial, thus taking care of (a).

(b) $p=1$. We consider $L^{1}(G)$ embedded as a subspace in $M_{b}(G)$, the space of bounded Radon measures on $G$ with the topology as the dual of $C_{0}(G)$ (the space of continuous functions on $G$ that vanish at infinity). Denoting by $\mu_{g}$ the measure generated by $g \in L^{1}(G)$, we have that the ball $\left\{\mu_{g} \in M_{b}(G)\right.$ : $\left.\left\|\mu_{g}\right\| \leq M\right\}$ is weak ${ }^{*}$-compact in $M_{b}(G)$, and hence there exists $\mu \in M_{b}(G)$ and a subsequence $\left(t_{m(k)}\right)$ of $\left(t_{m}\right)$ for which $\mu_{t_{m}(k)}(h) \rightarrow \mu(h)$ for all $h \epsilon$ $C_{0}(G)$. An argument similar to that in the second paragraph of (a) gives that $\operatorname{supp}(\hat{\mu}) \subset \boldsymbol{r}_{n}$.

Now, from [3, (31.7) and (23.10)], $\hat{\mu}=\hat{\mu} \hat{k}_{n}$, where $k_{n}=\lambda\left(V_{n}\right)^{-1} \xi_{V_{n}}$; we deduce from the fact that the Fourier transform is one-to-one that $\mu=\mu_{t}$ for some $t \in L^{1}(G)$. The remainder of the proof now follows as in (a)。

3. Characterisations of $\operatorname{Lip}_{p} \alpha$. The first result we require is the following analogue of Bernstein's theorem:

Theorem 1. If, for some $\alpha>0, E_{n}(p ; f)=O\left(\beta_{n+1}^{\alpha}\right)$, then

$$
\omega(p ; f ; \delta)= \begin{cases}O\left(\delta^{\alpha}\right), & 0<\alpha<1, \\ O(\delta|\log \delta|), & a=1, \\ O(\delta), & a>1 .\end{cases}
$$

Proof. For each $n \in\{1,2, \ldots\}$ we have (see the preceding Lemma) the existence of $t_{n}^{*} \in L_{\Upsilon_{n}}^{p}(G)$ for which $E_{n}(p ; f)=\left\|f-t_{n}^{*}\right\|_{p}$. By assumption 
$\left\|f-t_{n}^{*}\right\|_{p} \leq B \beta_{n+1}^{a}$ for some constant $B>0$. Define

$$
s_{1}=t_{1}^{*} ; \quad s_{n}=t_{n}^{*}-t_{n-1}^{*} \quad(n \in\{2,3, \ldots\}) .
$$

Then

$$
\left\|s_{n}\right\|_{p} \leq\left\|t_{n}^{*}-f\right\|_{p}+\left\|f-t_{n-1}^{*}\right\|_{p} \leq B\left(\beta_{n+1}^{a}+\beta_{n}^{\alpha}\right) \leq 2 B \beta_{n}^{a} \quad(n \in\{2,3, \ldots\}) .
$$

Thus we can find $C>0$ such that for all $n \in\{1,2, \ldots\}$,

$$
\left\|s_{n}\right\|_{p} \leq C \beta_{n}^{\alpha} \text {. }
$$

Now $\sum_{k=1}^{n} s_{k}=t_{n}^{*}$ converges in $L^{p}(G)$ to $f$ as $n \rightarrow \infty$. Hence, for any $a \in G, r_{a}\left(\sum_{k=1}^{n} s_{k}\right)-\sum_{k=1}^{n} s_{k}$ converges in $L^{p}(G)$ to $\tau_{a} f-f$ as $n \rightarrow \infty$, and (iii)

$$
\left\|\tau_{a} f-f\right\|_{p} \leq \sum_{k=1}^{\infty}\left\|\tau_{a} s_{k}-s_{k}\right\|_{p} \leq \sum_{k=1}^{m}\left\|\tau_{a} s_{k}-s_{k}\right\|_{p}+2 \sum_{k=m+1}^{\infty}\left\|s_{k}\right\|_{p}
$$

$$
\leq 2 \sum_{k=1}^{m} \omega_{\mathbf{r}_{k}}(a)\left\|s_{k}\right\|_{p}+2 \sum_{k=m+1}^{\infty}\left\|s_{k}\right\|_{p}
$$

to make the last step we use [1, Theorem 1.3], which asserts that for $s \in$ $L_{\boldsymbol{\gamma}}^{p}(G)$ with $\mathbf{T}$ compact, and for a relatively compact open set $\nabla \subset \Gamma$ containing zero, we have

$$
\left\|\tau_{a} s-s\right\|_{p} \leq(\theta(\mathbf{T}+\nabla-\nabla) / \theta(\mathbf{T}))^{1 / 2}\left(\omega_{\nabla}(a)+\omega_{\mathbf{T}+\nabla-\nabla}(a)\right)\|s\|_{p},
$$

where $\theta$ denotes a Haar measure on $\Gamma$ chosen so that Plancherel's theorem holds, and $\omega_{\mathbf{r}}(a)=\sup \{|\gamma(a)-1|: \gamma \in \mathbf{T}\}$ (note that in (iii), $\boldsymbol{\Upsilon}_{k}$ is a compact open subgroup of $\Gamma)$. As $d(a, 0) \geq \beta_{k}$ for all $a \notin V_{k}$, we have for such $a$

$$
\omega_{\boldsymbol{r}_{k}}(a) \leq 2 \beta_{k}^{-1} d(a, 0) \text {. }
$$

But $\omega_{\mathbf{T}_{k}}(a)=0$ for $a \in V_{k}$ and so (iv) clearly holds for all $a \in G$. This, combined with (ii) and (iii), gives

$$
\omega(p ; f ; \delta) \leq 4 C \delta \sum_{k=1}^{m} \beta_{k}^{a-1}+2 C \sum_{k=m+1}^{\infty} \beta_{k}^{a}
$$

for any $\delta>0$.

Now suppose that $0<\delta \leq \beta_{1}$, and choose $m \geq 1$ so that $\beta_{m+1}<\delta \leq$ $\beta_{m}$. Then

$$
\begin{aligned}
\omega(p ; f ; \delta) & \leq 4 C \delta \sum_{k=1}^{m} \beta_{k}^{a-1}+2 C \delta^{a} \sum_{k=m+1}^{\infty}\left(\frac{\beta_{k}}{\beta_{m+1}}\right)^{a} \\
& \leq 4 C \delta \sum_{k=1}^{m} \beta_{k}^{a-1}+\frac{2 C}{1-\mu^{a}} \delta^{a}
\end{aligned}
$$


(recall that $0<\mu<1$ and $\beta_{n+1} \leq \mu \beta_{n}$ for all $n \in\{1,2, \ldots\}$ ). We now consider three cases according to whether $\alpha$ is smaller than, equal to, or greater than 1 .

(a) $0<a<1$. Since $\beta_{n+1} \leq \mu \beta_{n}$ for all $n \in\{1,2, \ldots\}$, we have

$$
\sum_{k=1}^{m} \beta_{k}^{\alpha-1} \leq \sum_{k=1}^{m} \mu^{(1-\alpha)(m-k)} \beta_{m}^{\alpha-1} \leq \frac{1}{1-\mu^{1-\alpha}} \delta^{\alpha-1} \text {. }
$$

Thus

$$
\omega(p ; f ; \delta) \leq \frac{4 C}{1-\mu^{1-\alpha}} \delta^{a}+\frac{2 C}{1-\mu^{a}} \delta^{\alpha}=O\left(\delta^{a}\right) .
$$

(b) $a=1$. Now

$$
\omega(p ; f ; \delta) \leq 4 C \delta m+2 C \delta /(1-\mu) .
$$

But $\delta \leq \beta_{m} \leq \mu^{m-1} \beta_{1}<1$ implies that

$$
m \leq\left(|\log \delta|+\left|\log \beta_{1}\right|\right) /|\log \mu|+1,
$$

whence the result follows.

(c) $a>1$. We have

$$
\omega(p ; f ; \delta) \leq\left(4 C \sum_{k=1}^{m} \beta_{k}^{a-1}+\frac{2 C}{1-\mu^{a}} \delta^{\alpha-1}\right) \delta=O(\delta)
$$

and the theorem is proved.

Theorem 1, together with the property $\Gamma=\bigcup_{n=1}^{\infty} \mathbf{r}_{n}$, guarantees us a rich supply of functions in $\operatorname{Lip}_{p} \alpha$ (at least in the case when $G$ is infinite).

If we have that each $V_{n}$ is actually a (compact open) subgroup of $G$, then the remarks in $[2, \mathrm{p} .64]$ show that

$$
E_{n}(p ; f) \leq \sup \left\{\left\|\tau_{a} f-f\right\|_{p}: a \in V_{n}\right\}
$$

for all $f \in L^{p}(G)$ (or, if $p=\infty$, for uniformly continuous $f$ ). In particular, if $f \in \operatorname{Lip}_{p} \alpha$ for some $\alpha>0$ and $\beta_{n+1}=\mu \beta_{n}$ for all $n \in\{1,2, \ldots\}$, then $E_{n}(p ; f)=O\left(\beta_{n+1}^{a}\right)$. This result combines with Theorem 1 to give (with $\beta_{n}$, $V_{n}$ as above)

Theorem 2. Let $a \in(0,1)$ be given. Then $f \in \operatorname{Lip}_{p} \alpha$ if and only if $E_{n}(p ; f)=O\left(\beta_{n+1}^{\alpha}\right)$ (where, for $p=\infty, f$ is taken to be uniformly continuous).

We can obtain more from [2, p. 64], namely that

$$
s_{n}(p ; f) \leq \sup \left\{\left\|\tau_{a} f-f\right\|_{p}: a \in V_{n}\right\}
$$


where $S_{n}(p ; f)=\left\|k_{n} * f-f\right\|_{p}, k_{n}=\lambda\left(V_{n}\right)^{-1} \xi_{V_{n}}$ and $\xi_{V_{n}}$ denotes the characteristic function of $V_{n}$. We then have (with $\beta_{n}, V_{n}$ as in Theorem 2)

Theorem 3. Let $\alpha \in(0,1)$ be given. Then $f \in \operatorname{Lip}_{p} \alpha$ if and only if $s_{n}(p ; f)=O\left(\beta_{n+1}^{a}\right)$ (where, for $p=\infty, f$ is taken to be uniformly continuous).

Note that if $G$ is taken to be compact in Theorem 3, then each $k_{n} * f$ is just a partial sum of the Fourier series of $f$.

Theorems 2 and 3 require that each $V_{n}$ be a subgroup of $G$. This happens, for example, if we have a neighbourhood basis $\left(V_{n}\right)$ at zero consisting of a strictly-decreasing sequence of compact open subgroups of $G$ (such a neighbourhood basis always exists in an infinite first-countable 0-dimensional locally compact group; see $[3,(7.7)])$, any strictly-decreasing sequence $\left(\beta_{n}\right)$ of positive numbers, and a translation-invariant metric $d$ defined by

$$
d(x, y)= \begin{cases}\beta_{n+1}, & x-y \in V_{n} \backslash V_{n+1}, \\ \beta_{1}, & x-y \notin V_{1}, \\ 0, & x=y,\end{cases}
$$

(cf. $[4, \$ 2]$ ). In this case Theorems 2 and 3 are valid provided that there exists $\mu \in(0,1)$ such that $\beta_{n+1} \leq \mu \beta_{n}$ for all $n \in\{1,2, \ldots\}$.

\section{REFERENCES}

1. Walter R. Bloom, Bernstein's inequality for locally compact Abelian groups, J. Austral. Math. Soc. 17 (1974), 88-101.

2. - Jackson's Theorem for locally compact abelian groups, Bull. Austral. Math. Soc. 10 (1974), 59-66.

3. Edwin Hewitt and Kenneth A. Ross, Abstract harmonic analysis. Vols. I, II, Die Grundlehren der math. Wissenschaften, Bände 115, 152, Springer-Verlag, Berlin; Academic Press, New York, 1963, 1970. MR 28 \#158; 41 \#7378; erratum, 42, p. 1825 .

4. P. L. Walker, Lipschitz classes on 0-dimensional groups, Proc. Cambridge Philos. Soc. 63 (1967), 923-928. MR 35 \#7081.

5. A. Zygmund, Trigonometric series. Vols. I, II, 2nd ed., Cambridge Univ. Press, New York, 1968. MR 38 \#4882.

DEPARTMENT OF MATHEMATICS, THE UNIVERSITY OF TASMANIA, HOBART, TASMANIA, AUSTRALIA

Current address: School of Mathematical and Physical Sciences, Murdoch University, Murdoch, Western Australia, Australia 